\title{
COO-Transactions: Supporting Cooperative Work
}

\author{
Pascal Molli \\ Bâtiment LORIA, Campus scientifique \\ B.P. 239, 54506 Vandœuvre-lès-Nancy CEDEX \\ molli@loria.fr \\ CRIN/CNRS - Université Henri Poincaré, NANCY I
}

\begin{abstract}
In $C O O$, cooperation is a problem of synchronization among development activities running in parallel. We propose to encapsulate development activities within COO-transactions to ensure general properties on their concurrent execution. We have developed a new correctness criterion called COO-serializability which ensures consistency property in a cooperative context. COO-serializability is purely syntactic criterion based on the log analysis of repository access. In this paper, we describe COO-serializability foundation and how to evaluate it incrementally using the COO-protocol. Finally, we describe how we have implemented the $C O O$-protocol in the $C O O$ environment.
\end{abstract}

Keywords: Cooperation, Concurrency control, COO-serializability.

\section{Introduction}

In a natural way, when several users work together towards a common goal, they cooperate. To handle real development, a software engineering environment must allow cooperation between all actors of the development.

It is quite hard to characterize cooperation because there are many ways to cooperate. In Adèle [2] or EPOS [6], cooperation is a problem of change propagation between workspaces. In Marvel [1] or Merlin [14], cooperation is a problem of coordination of operations issued by users. Thus, in all cases cooperation occurs when users work together on a shared set of objects.

In COO $[11,12]$, cooperation is a problem of synchronization between development activities. We propose to encapsulate development activities into COOtransactions to handle problems of concurrent execution of development activities and to preserve data consistency.

COO-transactions support the cooperative nature of development activities by allowing data exchange during their execution. By this way, we relax the Isolation property generally fulfilled by traditionnal transactions but we ensure the others properties: Atomicity, Consistency and Durability [13]. 
To achieve this goal, we have built a new correctness criterion called COOserializability. This criterion can be seen as an extension of serializability to handle cooperative executions. COO-serializability can be verified by just analyzing the access log to the repository. Our approach is completely syntactic in opposition with semantic approach as Transaction Group [19].

In this paper we present the foundation of COO-serializability and how to evaluate it incrementally using the COO-protocol. Finally, we describe the implementation and the general behavior of COO-transactions.

\section{Development activities}

Schematically, a software development can be break down into development activities. A user works within a development activity (see figure 1).

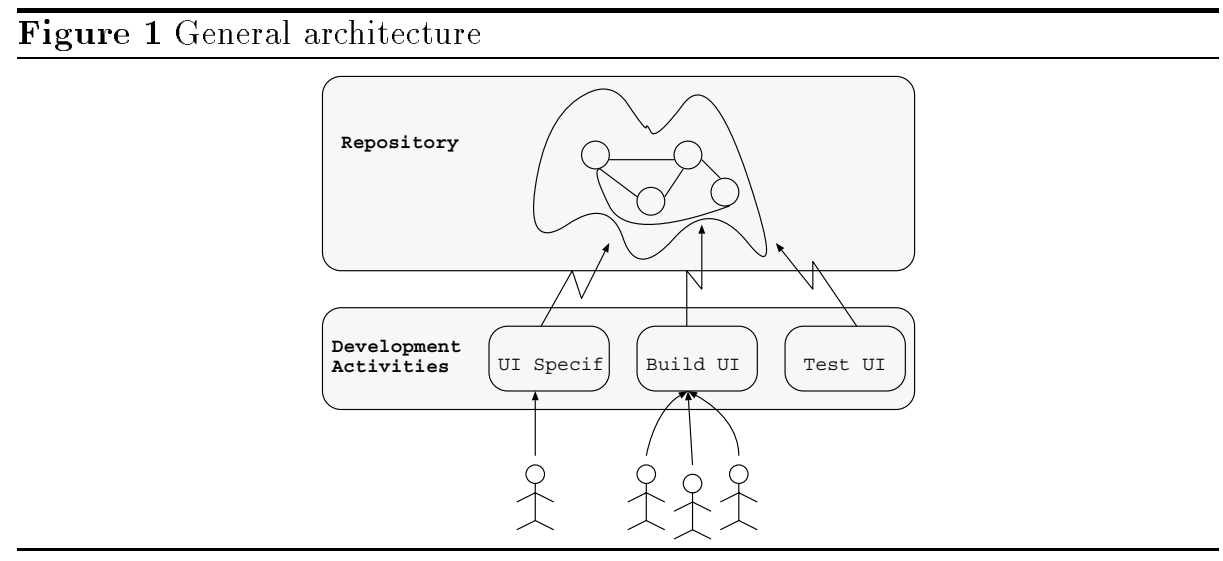

A development activity is goal-oriented: for example "build a library". Users work within the context of an activity and build interactively its "program" by retrieving objects from the repository and calling tools on local versions of these objects. In the example below, we represent the execution of the activity $A_{0}$ which builds a library lib starting from its specification spec:

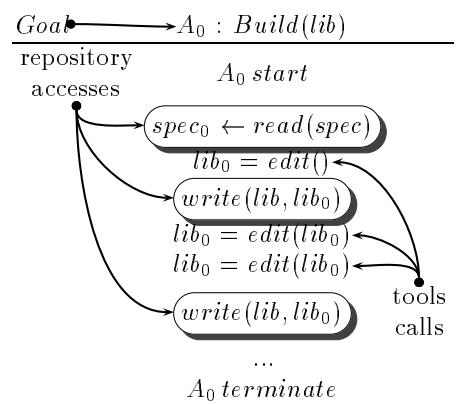


1. Users build the context of the activity: spec $_{0} \leftarrow \operatorname{read}($ spec $)$ means that a local object spec $_{0}$ is created in the context of $A_{0}$ with the value of the object spec stored in the repository.

2. Users call tools to create or transform local objects: lib $b_{0}=e d i t()$ or $l i b_{0}=$ edit $\left(l i b_{0}\right)$.

3. Users update objects in the repository: write $\left(l i b, l i b_{0}\right)$ means that the value of $l i b$ in the repository is updated with the value of the local object $l i b_{0}$.

We can see the repository like a shared memory accessed cooperatively by development activities. The problem is to prevent concurrent accesses to corrupt this memory.

In $\mathrm{COO}$, we encapsulate such activities in COO-transactions to preserve activities from bad behavior like lost update or dirty reads [13]. Hereafter, we give a motivating example to show what we expect from $\mathrm{COO}$-transactions.

\section{Motivating Example}

Suppose two activities of documentation updating the same document at the same time. Each activity works on its own copy of the document, makes some changes and releases an intermediate version of the document to be used by the other one.

The following execution scenarii illustrate possible synchronization problems between these two activities.

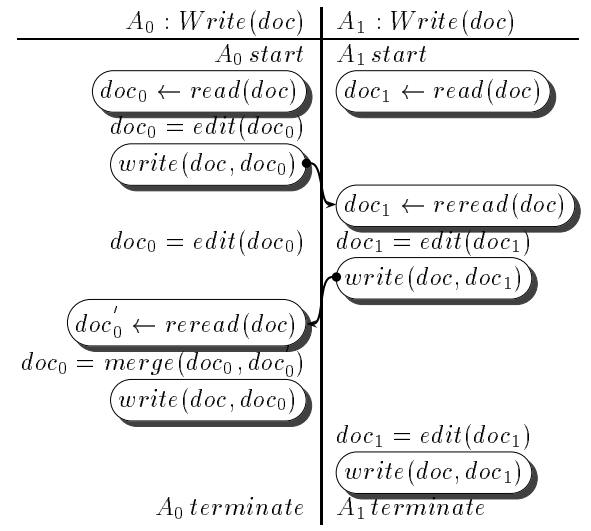

In the execution described above, notice that:

- cooperation occurs through data exchange between $A_{0}$ and $A_{1}$ (as highlighted by arrow on figure above).

- This kind of cooperation potentially leads to a merging stage, but merge operations is done inside a single activity without concurrency problems. From the point of view of activities synchronization, a merge operation is similar to an edit operation. 
- The last update of $A_{0}$ is overridden by the last update of $A_{1}$. COO-transactions must prevent such lost updates.

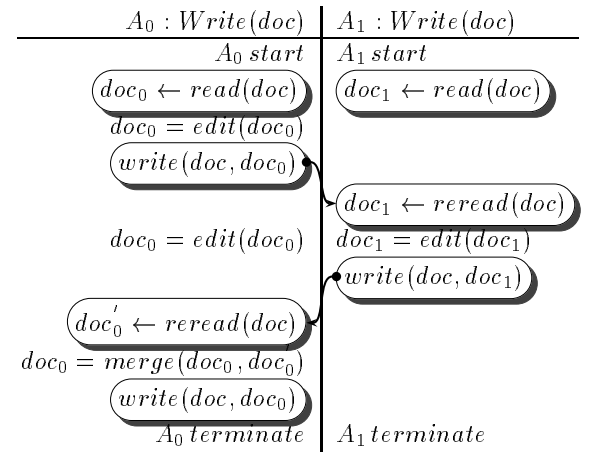

The figure above illustrate another problem. The final document produced by the execution of $A_{0}, A_{1}$ has not been reread by $A_{1}$. Typically, the result of cooperation between $A_{0}$ and $A_{1}$ has not been reviewed by an actor of this cooperation. We say that $A_{0}$ and $A_{1}$ have not converged to an unique value of the document. This scenario is dangerous because $A_{1}$ commits with a outdated view of document contents. We want that COO-transactions avoid such scenario.

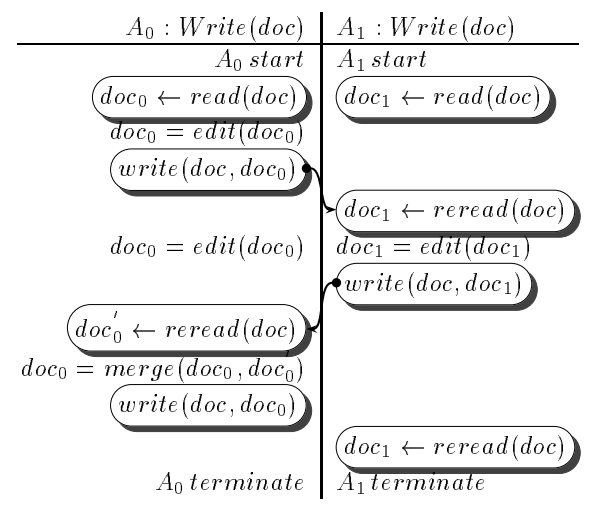

The last execution described above shows a correct execution. The two activities has converged on a single value of documentation before committing. We want that COO-transaction force users to produce this kind of execution.

We now describe how COO-serializability can determine correct execution by just analyzing the access log.

\section{COO-Serializability}

The COO-serializability [5] ensures that, even a COO-transaction reads intermediate results of another active COO-transaction, when it commits, all data inside it context are produced by committed COO-transactions. This allows users to start working with early drafts produced by others COO-transactions and the 
COO-protocol will force reread operations to ensure that new configuration will be builded from consistent data. If users, by themselves, don't introduce inconsistencies, then the new states produced by COO-transactions will be consistent.

COO-serializability is based on the log-analysis of repository access, so it is a purely syntactic criterion. Suppose that the two transactions $t_{0}, t_{1}$, working on two objects lib, app: $t_{0}$ codes the library $l i b$ and $t_{1}$ uses lib for coding the application $a p p$. From the point of view of repository access, we can observe the following log:

\begin{tabular}{r|l}
$t_{0}:$ Build $(l i b)$ & $t_{1}:$ Build $($ App $)$ \\
\hline lib $\leftarrow$ read $(l i b)$ & $a p p_{1} \leftarrow \operatorname{read}(a p p)$ \\
write $\left(l i b, l i b_{0}\right)$ & \\
& $l i b_{1} \leftarrow \operatorname{read}(l i b)$ \\
write $\left(l i b, l i b_{0}\right)$ & write $\left(\right.$ app, app $\left.p_{1}\right)$ \\
write $\left(l i b, l i b_{0}\right)$ & \\
$t_{0}$ commit & \\
& $\begin{array}{l}\text { lib } b_{1} \leftarrow \operatorname{read}(l i b) \\
\text { write }\left(a p p, a p p_{1}\right) \\
\\
t_{1} \text { commit }\end{array}$
\end{tabular}

In this $\log , t_{0}$ and $t_{1}$ cooperate because $t_{1}$ reads $l i b$ while $t_{0}$ is active. Next, $t_{1}$ rereads $l i b$ produced by $t_{0}$ which is committed. In this $\log t_{0}$ and $t_{1}$ commit with only committed data in their contexts. Thus, COO-serializability must accept this $\log$.

\section{COO-Serializability Foundation}

COO-serializability is based on the following observation: if cooperation helps to determine the value of committed data, from the point of view of the neteffect on the repository, some data exchange can be ignored. This is possible to carefully rewrite the log by hiding operations related to the cooperation process itself and extracting a sub-log that we call useful sub-log. At the opposite of the complete log, it makes sense to test serializability of the useful sub-log to determine the correctness of the original log. We have determined two rules for extracting useful sub-log from a cooperative log:

Last Occurrence Rule Cooperation leads to repeat read/write operations occurrences on an object: we obtain a sequence of identical operations on the same object. In our example, $t_{0}$ repeats three times write $\left(l i b, l_{i} b_{0}\right)$. Assuming that transactions only commit consistent values, the last occurrence of each operation belongs to the useful sub-log.

We highlight on our example the application of this rule: 


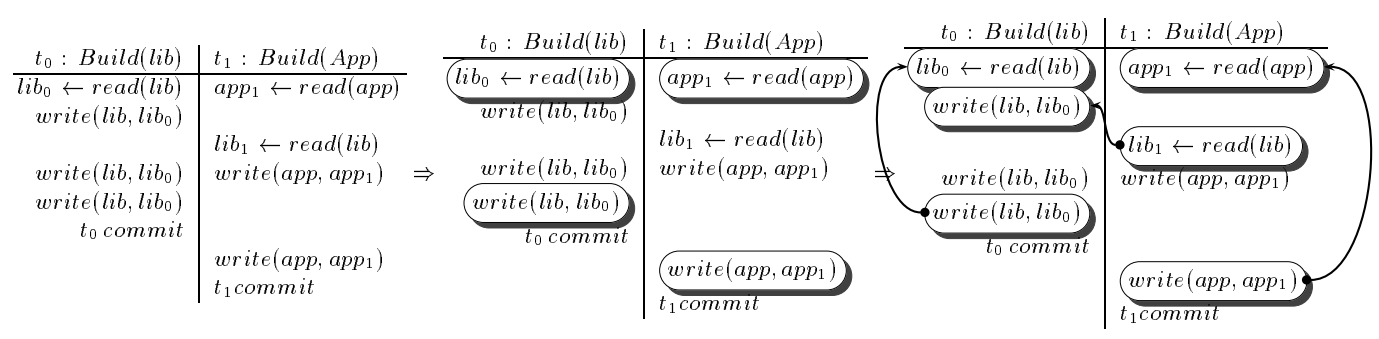

Fig. 1. A non-COO-serializable execution

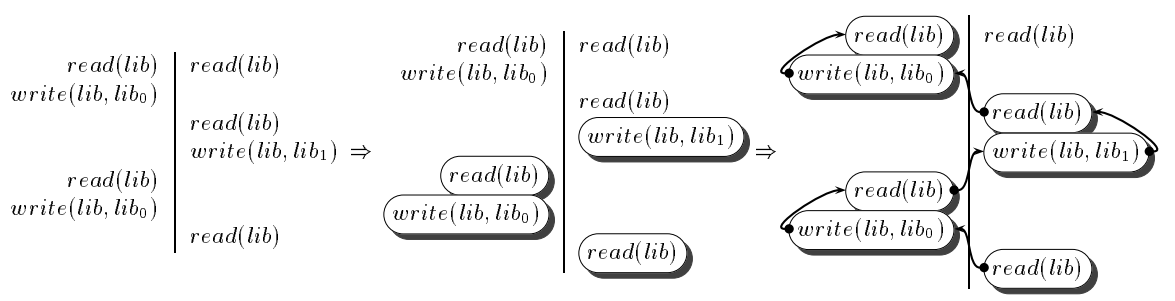

Fig. 2. CS-serializability with mutual inconsistent read

We illustrate this case on the figure 2. The useful sub-log selected by the Last Occurrence and Causal Propagation rules is nearly identical to the entire $\log$. The useful sub-log is not serializable and thus, the original log is not COOserializable. However, this kind of log is very representative of cooperation and must be accepted.

Cooperative serializability $[17,16]$ solves this problem by grouping transactions. All conflicts occuring between transactions belonging to the same group are ignored, but group of transactions must be serializable.

This approach is interesting but presents two major drawbacks: firstly the group of transactions must be declared before to start the execution, secondly there is no control within a group of transactions. We propose to enhance COOserializability with the notion of group of transactions but we want to detect group dynamically and control conflicts inside a group of transactions. This leads us to add two additional rules to COO-serializability:

Group Convergence Rule First, we focus on control inside a group of transaction. We think that when two users cooperate to determine the values of some objects, they must converge to an unique value for each object before committing their transactions. For example, if two transactions modify the same library they must converge to an unique value of this library before committing. Clearly, we don't want this log: 


$$
\begin{array}{r|l}
l i b_{0} \leftarrow \operatorname{read}(l i b) & l i b_{1} \leftarrow \operatorname{read}(l i b) \\
\text { write }\left(l i b, l i b_{0}\right) & \\
& \begin{array}{l}
\text { lib } b_{1} \leftarrow \operatorname{read}(l i b) \\
\text { write }\left(l i b, l i b_{1}\right)
\end{array} \\
\text { lib } b_{0} \leftarrow \operatorname{read}(l i b) & \\
\text { write }\left(\text { lib }, \text { lib } b_{0}\right) & \\
t_{0} \text { commit } & t_{1} \text { commit }
\end{array}
$$

because, $t_{1}$ commits without knowing the value of $l i b$ written by $t_{0}$. We want to force this log:

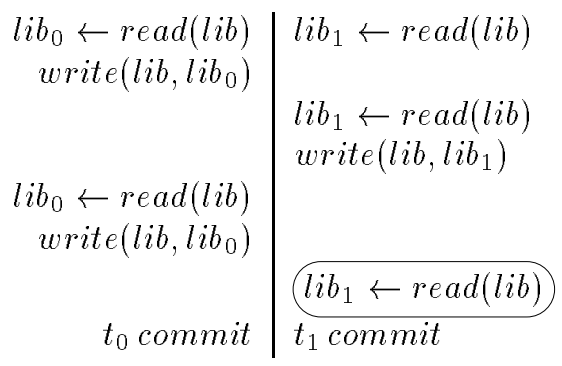

To achieve the convergence to an unique final state of a group of transactions, we require that the last value produced by a transaction in the group to be read by all the others.

Group Condition Rule We focus now on the second drawback: determining the group of transactions dynamically. Intuitively, transactions are grouped if there are mutual reading of their intermediate results. We can easily detect if a log contains such characteristics. We take the following convention: if a transaction $t_{i}$ reads an object $x$ produced by a transaction $t_{j}$, we have a relation $t_{i} \mathcal{I} \mathcal{R} t_{j}$. Let $\mathcal{I} \mathcal{R}^{*}$ the transitive closure of $\mathcal{I} \mathcal{R}$. We can give now the Group Condition Rule:

$$
\begin{aligned}
& \forall t_{i}, t_{j}, \text { if } t_{i} \mathcal{I} \mathcal{R}^{*} t_{j} \wedge t_{j} \mathcal{I} \mathcal{R}^{*} t_{i} \\
& \Rightarrow t_{i}, t_{j} \text { are grouped }
\end{aligned}
$$

We illustrate the Group Condition and Group convergence rule on figure 3 . In this log, $t_{0}$ works on a library $l i b$ while $t_{1}, t_{2}$ use this library to code the application app. The sequence:

\begin{tabular}{r|l}
$t_{1}$ & $t_{2}$ \\
$\ldots$ & $\ldots$ \\
write $(a p p)$ & \\
& $\begin{array}{l}\operatorname{read}(a p p) \\
\text { write }(a p p)\end{array}$ \\
$\operatorname{read}(a p p)$ & \\
$\ldots$ & $\ldots$
\end{tabular}

activates the Group Condition Rule, so $\left\{t_{1}, t_{2}\right\}$ are grouped. Thus, the execution of $\left\{t_{1}, t_{2}\right\}$ must satisfy the Group Convergence Rule and the $\left\{t_{0}\right\},\left\{t_{1}, t_{2}\right\}$ must be COO-serializable. 
Conflict occuring within $\left\{t_{1}, t_{2}\right\}$ are ignored so the useful sub-log determined on $\left\{t_{0}\right\},\left\{t_{1}, t_{2}\right\}$ is serializable. Within $\left\{t_{1}, t_{2}\right\}$, the last value produced by $t_{1}$ on app has been read by $t_{2}$ and reciprocally, so the Group Convergence Rule is satisfied. This $\log$ is COO-serializable.

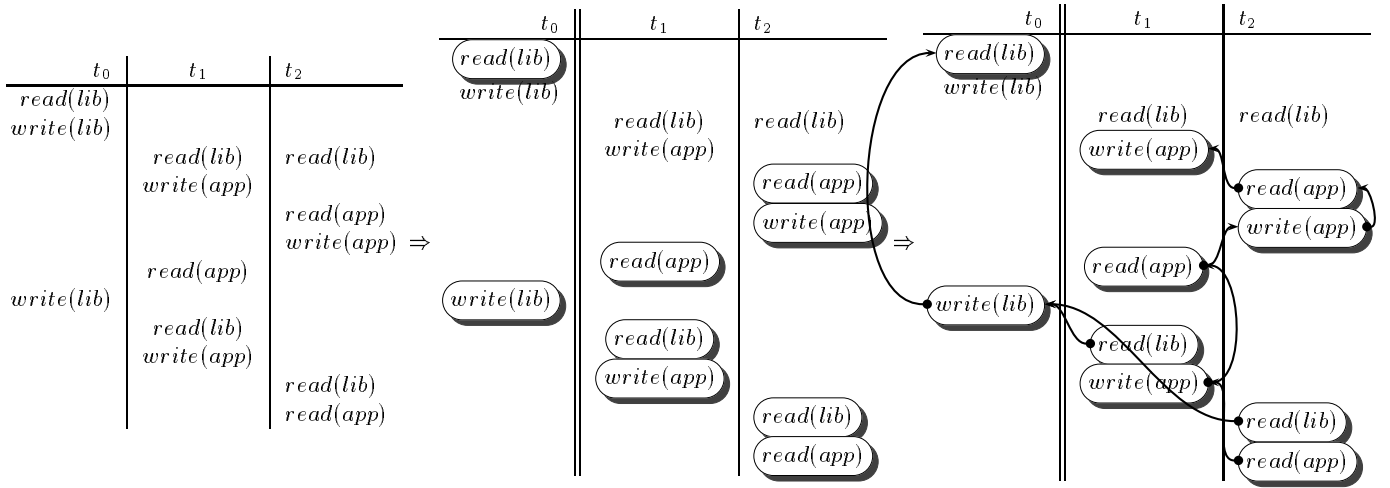

Fig. 3. A COO-serializable execution with $\left\{t_{1}, t_{2}\right\}$ in the same group

\section{Recovery strategy}

Of course, breaking down isolation like we do in COO-Serializability causes serious problems for recovery. However, we can verify that COO-serializability is a prefix commit-closed property [3]. So, COO-serializable logs are recoverable, but it is not possible to avoid cascading aborts.

Indeed, COO-serializability forces each transactions to commit after the commitment of all transactions from which it reads uncommitted data. This means that every recovery strategy based on rollback leads to propagate aborts to other transactions, but only to uncommitted transactions.

To resume, it's possible to abort COO-transactions and rollback to an initial state, but it is not realist in the context of development activities.

Advanced transaction models like [20][split and join transaction] or [24][contract model] propose instead of backward recovery approach (rollback), a forward or adaptative recovery approach. In our context, it means that an activity reaches its goal or it recovers a consistent state. Perhaps this state is not the initial state. We have built an extension of COO-transactions called Split-COOtransactions that extends COO-transactions with forward recovery support. This work is outside of the scope of this paper but, more details can be found in [23].

\section{COO-Protocol}

COO-serializability is very general correctness criterion which ensures consistency assuming that users work correctly. We present now a protocol which 
incrementally evaluates COO-serializability in the context of long transactions (as in the long transaction model).

To make the criterion evaluable, we introduce the notion of intermediate results. If a transaction releases an object and continues its execution then this object is considered as an intermediate object. Using the notion of intermediate result, we propose the following strategy to evaluate COO-serializability:

1. A result produced before the end of a transaction is an intermediate result. The user can call at any time the "upward_commit" operation to produce an intermediate result.

2. A result produced at the end of a transaction is a final result. All of the final results are produced in an atomic way during the execution of the "commit" operation:

$$
\begin{array}{|r|}
\hline t_{0} \text { commit } \\
\hline \text { write }\left(\text { app }, a p p_{0}\right) \\
\hline \text { write }\left(l i b, l i b_{0}\right) \\
\hline
\end{array}
$$

means that during the execution of the termination operation, both application and library are updated atomically in the repository.

3 . If a transaction produces an intermediate result, then it must produce the corresponding final result. The transaction remembers all objects that has been "upward_committed" and produces automatically the final results during its termination phase.

4. If a transaction reads an intermediate result of another transaction, then it must reread the corresponding final result. If a transaction $t_{1}$ reads an intermediate result of an object $x$ produced by a transaction $t_{0}$ then a dependency $t_{1} \stackrel{x}{\rightarrow} t_{0}$ is created. If the transaction $t_{1}$ reads a result of $x$ and $t_{0}$ is committed, then the dependency is removed $\left(t_{1} \stackrel{x}{\leftrightarrow} t_{0}\right)$. We illustrate this mechanism in the figure below:

\begin{tabular}{|c|c|}
\hline$t_{0}:$ Build $(l i b)$ & $t_{1}:$ Build (App) \\
\hline$\left(i b_{0} \leftarrow \operatorname{read}(l i b)\right)$ & $\left(a p p_{1} \leftarrow \operatorname{read}(a p p)\right)$ \\
\hline$I z b_{0}=\operatorname{dit}\left(t i b_{0}\right)$ & \\
\hline upward_commit (lib,libo) & $t_{1} \stackrel{\operatorname{lib}}{\longrightarrow} t_{0}$ \\
\hline$l i b_{0}=\operatorname{edit}\left(l i b_{0}\right)$ & $>(\underbrace{(\operatorname{li} b))}_{a p p_{1}=\operatorname{lid} b_{1} \leftarrow \operatorname{read}\left(a p p_{1}\right)}$ \\
\hline upward_commit $\left(l i b, l i b_{0}\right)$ & upward_commit $\left(a p p, a p p_{1}\right)$ \\
\hline$I i b_{0}=\operatorname{edit}\left(I i b_{0}\right)$ & \\
\hline$t_{0}$ commit & \\
\hline (write $\left.\left(l i b, l i b_{0}\right)\right)$ & \\
\hline & $t_{1}$ commit \\
\hline & $\rightsquigarrow$ error \\
\hline
\end{tabular}

The above cooperative $\log$ of $t_{0}, t_{1}$ is not correct. In fact, $t_{1}$ reads an intermediate result from $t_{0}\left(t_{1} \stackrel{l i b}{\rightarrow} t_{0}\right)$, it tries to commit without taking into account the final result of lib. Then, the "commit" operation itself is refused and the execution of $t_{1}$ continues. If the user decides to reread the final result of $l i b$, then the transaction is allowed to commit: 


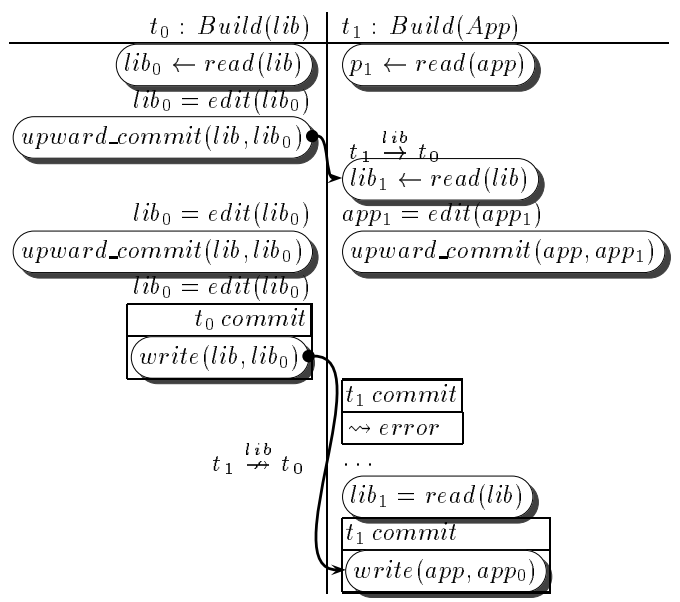

The previous strategy allows to maintain the Last Occurrence and Causal Propagation Rules. The Group Condition Rule can be verified easily: if there is a cyclic dependency, then all transactions involved in the cycle are grouped. We propose the following group commit strategy in order to evaluate the Group Convergence Rule inside a group of transactions:

1. A transaction starts a group termination by trying to commit itself. By this action, it produces a set of potentially final results and becomes "ready to commit".

2. If another transaction of the same group tries to commit and all others transactions of the group are in the "ready to commit" state, then all transactions of the group commit simultaneously.

3. If another transaction of the same group tries to commit and all others transactions of the group are not in the "ready to commit" state, then it completes the set of potentially final results and becomes "ready to commit".

4. If a transaction of a group produces a new intermediate result, then the group commit tentative is aborted and all transactions of the group become "active". This is the way for a transaction to show its disagreement with the potentially new state of the repository. 


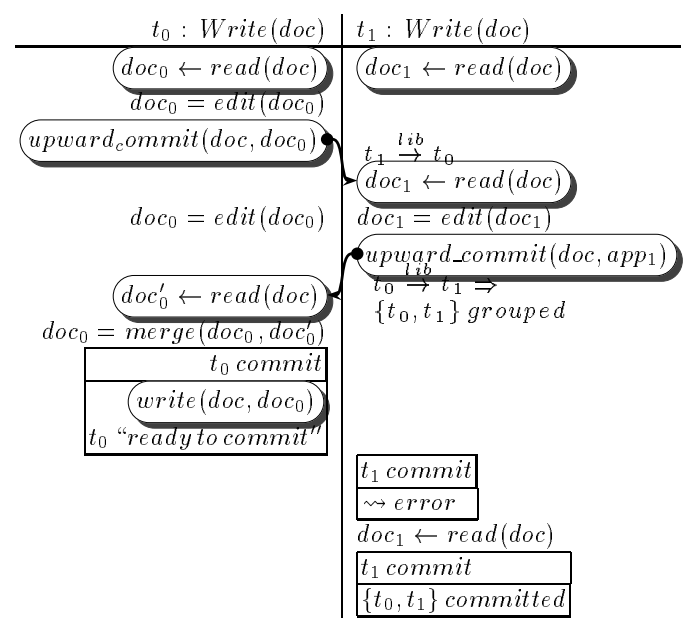

The COO-protocol detects that $\left\{t_{0}, t_{1}\right\}$ are grouped after $t_{0}$ reads an intermediate of $t_{1}$; there is a cyclic dependency $\left(t_{1} \stackrel{\text { lib }}{\rightarrow} t_{0}, t_{0} \stackrel{l i b}{\rightarrow} t_{1}\right)$. So $\left\{t_{0}, t_{1}\right\}$ must follow the group termination strategy in order to commit. $t_{0}$ initiates the group termination by trying to commit itself. $t_{0}$ writes a potentially final result of $d o c$ and becomes "ready to commit". The execution of $t_{0}$ is suspended. Next, $t_{1}$ tries to commit, the commit operation is refused since the potentially final state produced by $t_{0}$ has not been reread by $t_{1} . t_{1}$ continues its execution and rereads the documentation $(d o c), t_{0}$ agrees on this value by calling the commit operation. The potentially final result of $d o c$ is validated and the two transactions commit simultaneously.

To resume, the COO-protocol is only based on the incremental analysis of the $\log$ of read/write operations in the repository. This protocol ensures the COOserializability as described before. This protocol and its corresponding algorithms are formalized in VDM in [18].

\section{Implementation of the COO-Protocol}

We have implemented and experimented the COO-protocol in the COO-environment [12] with a support for nested long transactions. We have based the nested support on base/sub-base model as in $[8,15]$ [Damokles, Orion]. The base/sub-base model is emulated on a PCTE [9] repository extended by the P-RooT system [4] (see figure 4).

The behavior of the COO-system is very similar to the NSE [7] system. Users compose their object model using PCTE facilities then they launch long transactions from a common root long transaction in order to work on data.

Within the context of a long transaction, they can checkout an object (as the "acquire" command in NSE) to obtain a local copy and call a tool on it to change its value. All modifications of the copy are not visible for others transactions until users explicitely put the copy back to the parent transaction context.

The main difference with NSE is the way to make public a local modification. 


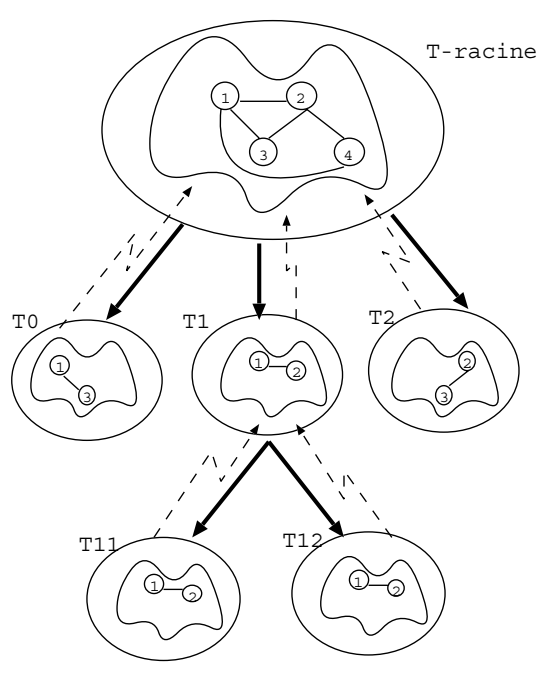

Fig. 4. COO-protocol in COO environment

Using NSE, there is an unique way: users call the "reconcile" operation to put back a copy in the repository. The local context of the transaction must be up-to-date with last state of the repository. If this condition is not satisfied, users must call the "resynch" command in order to be aware of the last value of object produced by parallel developments.

In COO, user can call:

1. the "upward-commit" operation to release an intermediate result of an object and the value doesn't need to satisfy constraints checking.

2. the "commit" operation to finish the transaction and make public all its modifications. Constraints checking is required on these changes.

If COO-serializability is violated, the COO-protocol will refuse the "commit" or "upward-commit" operation. In this case, the user needs to update his local context and continues its work in order to reestablish local consistency. Next, he can retry a "commit" or an "upward-commit" operation.

\section{Related Work}

Our approach of cooperation in software development environments is quite original in the domain. For software engineering environments like Adèle [2] or EPOS [6], cooperation is a problem of changes propagation between workspaces. In Adèle, users can choose a protocol for object sharing from a list of predefined strategies. The behavior of these protocols has been experimented and it is the responsibility of the user to choose the right protocol for the right situation. 
With the COO-protocol, users start the work in transactions with the warranty of producing coherent results if they do their job correctly.

The approach of cooperation in Marvel [1] or Merlin [14] is quite different. There is a collection of small transactions (typically a user calling a tool ...) synchronized by the software process. Problems of concurrent accesses occur only when two transactions really access the same object. In this case, Marvel or Merlin detects which transaction must be aborted. This approach cannot ensure general properties: transaction behavior must be explicitely described.

Concerning transaction models, our approach to support cooperation is quite original. In fact, models like transaction group [19] use a pure semantic correctness criterion, so it's not possible to ensure general properties to transactions.

Our work can be best compared with [22,21][Epsilon-serializability] in the sense that a transaction can read uncommitted data from another and so import inconsistency. Transaction can itself export inconsistency. The correctness of Epsilon-serializability is based on bounding the amount of imported and exported inconsistencies. Our approach is different in the sense that although we allow the import/export of inconsistency, we require convergence and final consistency.

Finally, we can compare our approach with Long Transaction model in configuration management $[10,7]$. Development activities are encapsulated in transactions with an optimistic concurrency control protocol. However, in this model, transactions do not ensure general properties.

\section{Conclusion}

We think that COO-serializability can improve teamwork support in configuration management tools. Cooperation is allowed and supported without preliminary declarations. Consistency is ensured by the COO-protocol that can be easily implemented. The burden on users is low. Sometimes, the COO-protocol will ask users to do several update before committing their changes.

Actually, $\mathrm{COO}$ is a research prototype and we plan a validation of our approach on a larger scale with a new prototype allowing real developments.

\section{References}

1. BARghouti, N. Supporting Cooperation in the MARVEL Process-Centered SDE. Proceedings of the Fifth ACM SIGSOFT Symposium on Software Development Environments 17, 5 (December 1992), 21-31.

2. Belkhatir, N., and Estublier, J. ADELE-TEMPO : An Environment to Support Process Modelling and Enaction. In Software Process Modelling and Technology, J. K. A. Finkelstein and B. Nuseibeh, Eds. Research Study Press, 1994.

3. Bernstein, P., Hadzilacos, V., and Goodman, N. Concurrency Control and Recovery in Database Systems. Addison-Wesly, 1987.

4. Canals, G., Charoy, F., Godart, C., and Molli, P. P-Root $6 \&$ COO: Extending PCTE with new Capabilities. In ICSE'94 - Workshop on Databases for Software Engineering (Sorrento, Italy, 1994), IEEE Press. 
5. Canals, G., Molli, P., and Godart, C. Concurrency control for cooperating software processes. In Proceedings of the 1996 Workshop on Advanced Transaction Models and Architecture (ATMA'96) (Goa, India, 1996).

6. ConRadi, R., AND AL. EPOS: Object-Oriented Cooperative Process Modelling. In Software Process Modelling and Technology, A. Finkelstein and J. Kramer and B. Nuseibeh, Ed. Research Study Press, 1994.

7. Courington, W. The Network Software Environment. Tech. Rep. FE197-0, Sum Microsystems Inc., February 1989.

8. Dittrich, K., Gotthard, W., and Lockemann, P. DamOKLES - A Database System for Software Engineering Environments, 1987.

9. ECMA. Portable Common Tool Environment (PCTE), Abstract Specification. Standard ecma-149, European Computer Manufacturers Association, June 1993.

10. Feiler, P., And Downey, G. Transaction-Oriented Configuration Management: A Case Study. Tech. Rep. CMU/SEI-90-TR-23 ESD-90/TR-224, Software Engineering Institute, Carnegie Mellon University, Pittsburgh, Pennsylvania 15213. November 1990.

11. Godart, C. COO: a Transaction Model to support COOperating software developers COOrdination. In 4th European Software Engineering Conference (ESEC4), Garmisch, LNCS 717 (1993).

12. Godart, C., Canals, G., Charoy, F., Molli, P., and Skaf, H. Designing and Implementing COO: Design Process, Architectural Style, Lessons Learned. In International Conference on Software Engineering (ICSE18) (1996). IEEE Press.

13. Gray, J., AND ReUter, A. Transaction Processing: Concepts and Techniques. Morgan Kaufmann, 1993.

14. Junkerman, G., Peushel, B., Schäfer, W., and Wolf, S. Merlin: Supporting Cooperation in Software Development Through a Knowledge Based Environment. In Software Process Modelling and Technology, J. K. A. Finkelstein and B. Nuseibeh, Eds. Research Study Press, 1994.

15. Kim, W., And Lochovsky, F., Eds. Object-Oriented Concepts, Databases and Applications. ACM Press, 1989.

16. K.Ramamritham, AnD Chrysanthis, P. In Search of Acceptability Criteria: Database Consistency Requirements and Transaction Correctness Properties. In Distributed Object Management, Özsu, Dayal, and Valduriez, Eds. Morgan Kauffman, 1993.

17. Martin, B., and Pedersen, C. Long-Lived Concurrent Activities. In Distributed Object Management, Özsu, Dayal, and Valduries, Eds. Morgan Kauffman, 1992.

18. Molli, P. Environnements de Développement Coopératifs. Thèse en informatique, Université de Nancy I - Centre de Recherche en Informatique de Nancy, 1996.

19. Nodine, M., Ramaswamy, S., And Zdonik, S. A Cooperative Transaction Model for Design Databases. In Database transaction models for advanced applications. A. Elmagarmid, Ed. Morgan Kauffman, 1992.

20. Pu, C., Kaiser, G., And Hutchinson, N. Split Transactions for Open-Ended Activities. In Proceedings of the 14 th international conference on VLDB (Los Angeles, September 1988), pp. 26-37.

21. Pu, C., AND LefF, A. Replica control in distributed systems: An asynchronous approach. In Proceedings of the 16th Annual ACM Conference on the Management of Data (Denver, May 1991), pp. 377-386.

22. Ramamritham, K., And Pu, C. A Formal Characterization of Epsilon Serialisability. IEEE Transactions on Knowledge and Data Engineering 7, 6 (December 1995), 997-1007. 
23. Skaf, H., Charoy, F., and Godart, C. A Hybrid Approach to Maintain Consistency of Cooperative Software Development Activities. In The Ninth International Conference on Software Engineering and Knowledge Engineering, SEKE'97 (Madrid, 1997).

24. Wachter, H., And Reuter, A. The ConTract Model. In Database Transaction Models for Advanced Applications, A. Elmagarmid, Ed. Morgan Kauffmann, 1992, ch. 7 , pp. 219-258. 